\title{
TWIST1/miR-584/TUSC2 pathway induces resistance to apoptosis in thyroid cancer cells
}

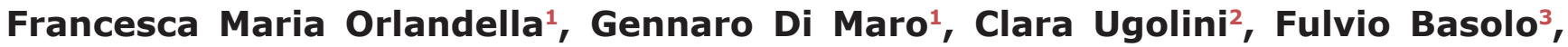 \\ Giuliana Salvatore ${ }^{1,4}$ \\ ${ }^{1}$ IRCCS SDN Spa, 80121 Napoli, Italy \\ ${ }^{2}$ Dipartimento di Area Medica, Azienda Ospedaliero-Universitaria Pisana, 56126 Pisa, Italy \\ ${ }^{3}$ Dipartimento di Patologia Chirugica, Medica, Molecolare e dell'Area Critica dell' Università di Pisa, 56126 Pisa, Italy \\ ${ }^{4}$ Dipartimento di Scienze Motorie e del Benessere, Universita' "Parthenope", 80133 Napoli, Italy \\ Correspondence to: Giuliana Salvatore, email: giuliana.salvatore@uniparthenope.it \\ Keywords: miR-584, TWIST1, TUSC2, anaplastic thyroid carcinoma, apoptosis \\ Received: February 23, $2016 \quad$ Accepted: September 02, 2016 Published: September 20, 2016
}

\section{ABSTRACT}

TWIST1, a transcription factor, plays a pivotal role in cancer initiation and progression. Anaplastic thyroid carcinoma (ATC) is one of the deadliest human malignancies; TWIST1 is overexpressed in ATC and increases thyroid cancer cell survival, migration and invasion. The molecular mechanisms underlying the effects of TWIST1 are partially known. Here, using miRNome profiling of papillary thyroid cancer cells (TPC-1) ectopically expressing TWIST1, we identified miR-584. We showed that TWIST1 directly binds miR-584 using chromatin immunoprecipitation. Importantly, miR-584 was up-regulated in human ATC compared to papillary thyroid carcinoma (PTC) and normal thyroid samples. Overexpression of miR-584 in TPC cells induced resistance to apoptosis, whereas stable transfection of anti-miR-584 in TPC-TWIST1 and $8505 \mathrm{C}$ cells increased the sensitivity to apoptosis. Using bioinformatics programs, we identified TUSC2 (tumor suppressor candidate 2) as a novel target of miR-584. TUSC2 mRNA and protein levels were decreased in TPC miR-584 and increased in TPC-TWIST1 anti-miR-584 cells. Luciferase assays demonstrated direct targeting. Restored expression of TUSC2 rescued the inhibition of apoptosis induced by miR584. Finally, qRT-PCR and immunohistochemical analysis showed that TUSC 2 was down-regulated in ATC and PTC samples compared to normal thyroids. In conclusion, our study identified a novel TWIST1/miR-584/TUSC2 pathway that plays a role in resistance to apoptosis of thyroid cancer cells.

\section{INTRODUCTION}

TWIST1 is a basic helix-loop-helix transcription factor essential for the development of mesoderm-derived tissues [1]. TWIST1 reactivation has been observed in many human cancers, where it was correlated with poor prognosis [2, 3]. TWIST1 promotes cancer progression [2] by inducing epithelial to mesenchymal transition (EMT) [4] and invadopodia formation [5]. TWIST1 promotes a cancer stem cell phenotype [6,7], inhibits apoptosis [8] and increases resistance to chemotherapy [2].

Thyroid carcinoma derived from follicular cells includes different malignancies, ranging from well-differentiated to undifferentiated (or anaplastic) carcinoma [9, 10]. The molecular mechanisms driving thyroid neoplastic progression are not fully understood [11]. Anaplastic thyroid carcinoma (ATC) is among the most lethal human cancers [12-17]. ATC cells show an infiltration of approximately 50\% tumor-associated macrophages, which increase tumor aggressiveness [18, 19]. EMT and stemness play an important role in the pathogenesis of ATC [20-22]. TWIST1 is up-regulated in ATC and increases cell migration, invasion and resistance to apoptosis [21, 23, 24].

Recently, we identified a set of mRNAs that mediates the biological effects of TWIST1 in thyroid 
cancer cells [25]. TWIST1 effects are also mediated by miRNAs [26-29]. miRNAs are a class of small nuclear RNA molecules that inhibit post-transcriptional gene expression. miRNAs have a central role in the establishment and progression of human cancers [30].

Here, using a miRNome screening analysis of papillary thyroid cancer cells (TPC-1, hereafter named TPC) ectopically expressing TWIST1 compared to control cells, we found that miR-584 was up-regulated by TWIST1, and we studied its role in thyroid cancer cells.

\section{RESULTS}

\section{TWIST1 alters the expression of a set of miRNAs in papillary thyroid cancer cells}

To identify miRNA targets of TWIST1, we carried out miRNome profiling of TPC cells transfected with TWIST1 compared to vector control cells. We found 45 miRNAs that were differentially expressed (14 upregulated and 31 down-regulated, Supplementary Tables $\mathrm{S} 1$ and S2) by more than 2-fold in TPC-TWIST1 cells.

To verify the microarray data, we performed qRT-PCR of the top 5 up-regulated and the top 5 downregulated miRNAs in TPC-TWIST1 cells. We confirmed $90 \%$ of the deregulated miRNAs (Figure 1); only miR-190 was not changed (data not shown). Among the most upregulated miRNAs, we focused on miR-584.
miR-584 is transcriptionally regulated by TWIST1

To determine whether TWIST1 directly binds miR-584, we performed chromatin immunoprecipitation (ChIP). TWIST1 binds to the E-box sequence motif (CANNTG) in its target genes [1]; thus we examined the 4-kb region upstream of miR-584 on chromosome $5 \mathrm{q} .32$ and found 14 E-boxes (Figure 2a). Chromatin samples of TPC-TWIST1 or control cells (pcDNA) were cross-linked and immunoprecipitated with anti-TWIST1 or with control (IgG1) antibodies. A region containing six consecutives E-boxes was amplified by qRT-PCR in TPC-TWIST1 cells but not in control cells (Figure 2b). No amplification was obtained with anti-IgG1 precipitates (Figure 2b) or when primers for the control GAPDH promoter were used (Figure 2c).

\section{miR-584 is up-regulated in human ATC samples}

To study the in vivo expression of miR-584, we measured miR-584 levels using qRT-PCR in 10 normal thyroid (NT), 11 PTC, and 6 ATC tissues samples. As shown in Figure 3, most of the ATC ( 80\%) samples overexpressed ( 3 -fold) miR-584 compared to the NT and PTC samples.

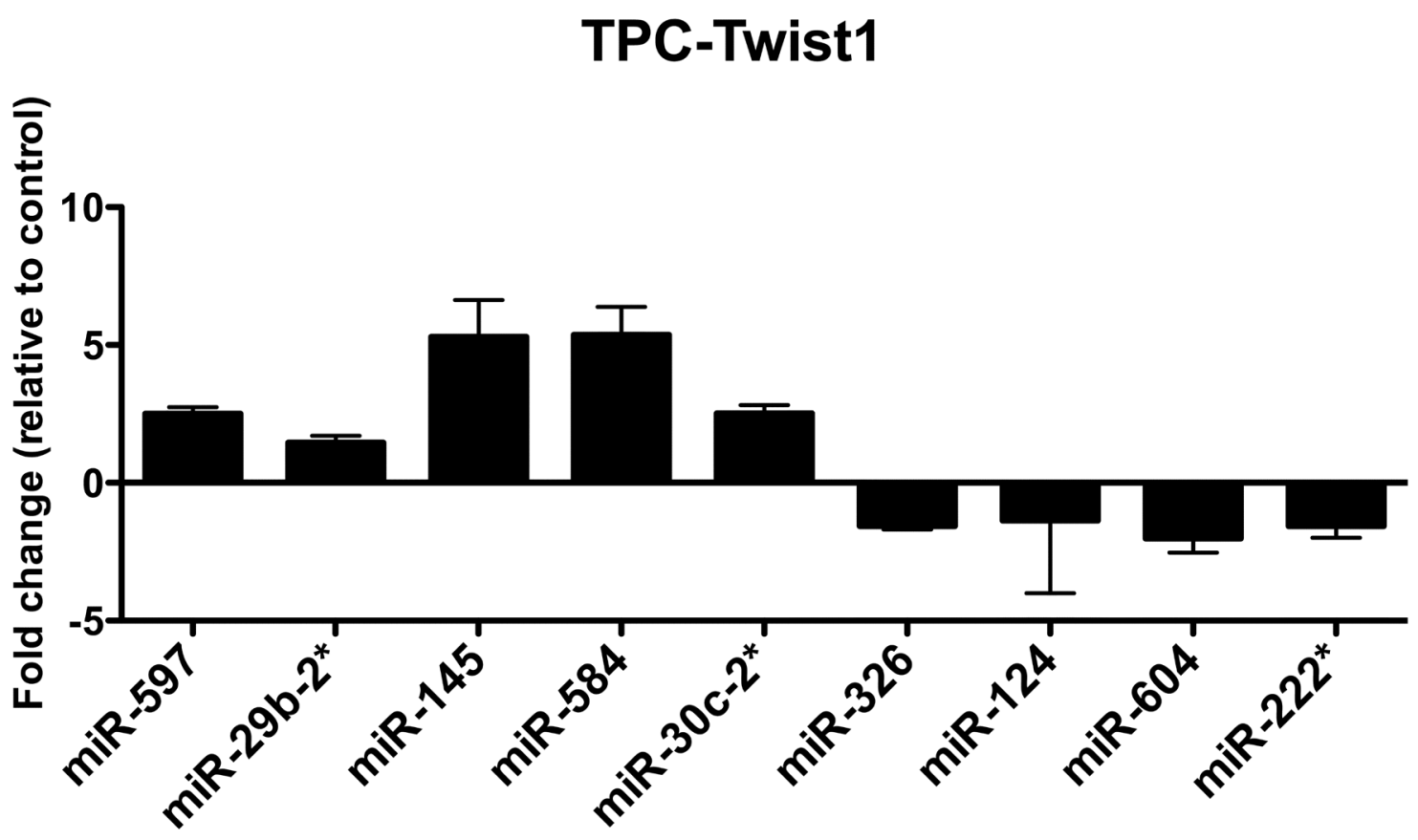

Figure 1: Expression levels of the indicated miRNAs in TPC-TWIST1 cells. qRT-PCR analysis of the indicated miRNAs in TPC-TWIST1 cells compared to vector control cells. The expression level of each miRNA was normalized to the level of U6 snRNA, and fold changes were evaluated using the $2^{-\Delta \mathrm{Ct}}$ method, assuming that the value of the vector control cells is equal to 1 . The average results of three independent experiments are reported with error bars indicating standard deviation. 


\section{Forced expression of miR-584 does not impair migration, invasion and proliferation of TPC cells}

TWIST1 sustains the invasive and migratory phenotype of thyroid cancer cells $[21,23]$; therefore, we investigated whether miR-584 was in part responsible for these effects. Thus, we stably transfected TPC cells, in which the basal level of miR-584 is shown in Figure $\mathrm{S} 1 \mathrm{a}$, with a precursor of miR-584 or with an empty vector (miR-null). qRT-PCR confirmed that miR-584 was increased in a selected mass population (Figure $\mathrm{S} 1 \mathrm{~b}$ ).

Next, we performed wound-healing assays in TPC miR-584 or TPC miR-null cells and monitored cell migration after 12 and 24 hours. As shown in Figure S2a, miR-584 did not influence the motility of TPC cells as both cell lines showed equal wound healing. We also performed a Matrigel invasion assay in which TPC miR-584 and control cells were seeded into the top chamber of transwells, and their ability to invade into the Matrigel was evaluated after 24 hours. As shown in Figures S2b and S2c, the TPC miR-584 and control cells had similar cell invasion abilities. Finally, we assessed whether miR-584 influenced cell proliferation rate. As shown in Figure S1d, both cell lines grew at a similar rate.

\section{miR-584 protects TPC cells from apoptosis induced by doxorubicin and staurosporine}

We investigated whether miR-584 affects the sensitivity to apoptosis because TWIST1 is an anti-apoptotic factor $[1,2]$. TPC miR-584 and control cells were treated with different doses of doxorubicin or staurosporine and counted after 48 and 24 hours, respectively. The $50 \%$ growth inhibitory concentration $\left(\mathrm{IC}_{50}\right.$ ) of doxorubicin in TPC miRnull cells was $8.9 \mathrm{nM}$, while in TPC miR-584 cells, it was $48 \mathrm{nM}$ (Figure 4a, left). Similarly, the $\mathrm{IC}_{50}$ of staurosporine in TPC miR-null cells was $123 \mathrm{nM}$, while in TPC miR-584 cells, it was $334 \mathrm{nM}$ (Figure 4a, right).

To further confirm these findings, we treated TPC miR-584 and TPC miR-null cells with doxorubicin and staurosporine at $\mathrm{IC}_{50}$ values, stained them with 7AAD/ Annexin $\mathrm{V}$ and analyzed the cells by flow cytometry. As shown in Figure 4b, the percentage of apoptotic cells (P3) in TPC miR-584 cells treated with doxorubicin was $8.1 \%$, while in TPC miR-null cells, it was 19\%. The percentage of apoptotic cells (P3) in TPC miR-584 cells treated with staurosporine was $7.6 \%$, while in TPC miR-null cells, it was $20.3 \%$.

Accordingly, TPC miR-584 cells showed decreased activation of cleaved CASPASE 3 compared to control cells following the treatments (Figure 4c). Overall, these results demonstrate that miR-584 protects TPC cells from apoptosis.

$\mathbf{a}$

Location: $5 q 32$

Chr 5:149062313- 149062409

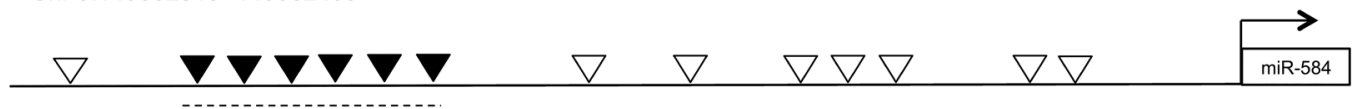

b

C
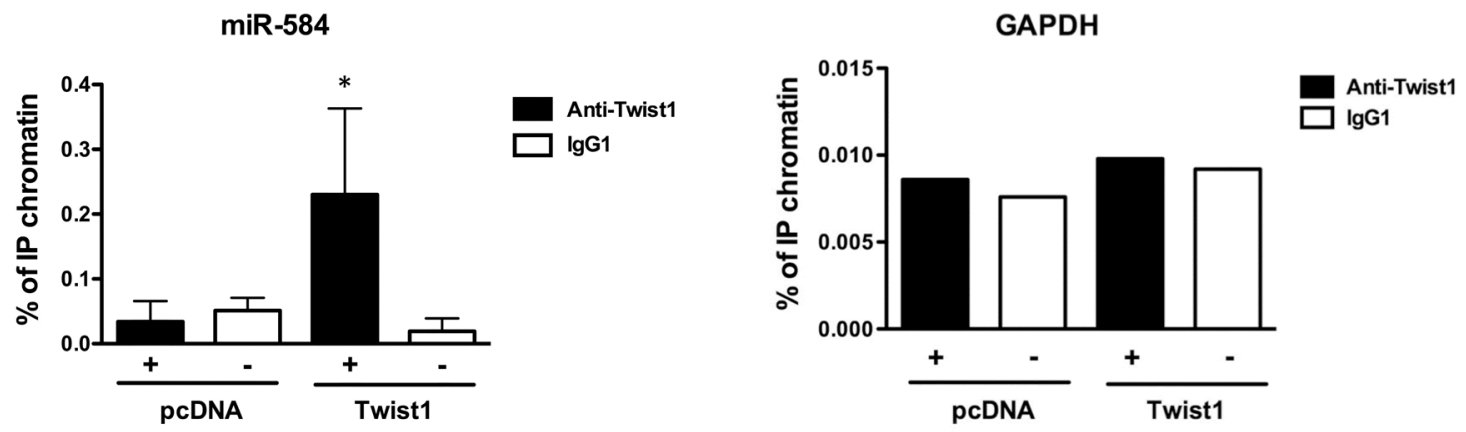

Figure 2: TWIST1 directly binds a region upstream of miR-584. a. Schematic representation of all E-box binding sites (indicated as -) in the genomic locus of miR-584. Black $\boldsymbol{\nabla}$ indicate the E-boxes amplified in ChIP assays. b. ChIP assay, followed by qRT-PCR, was performed with TPC-TWIST1 or control (pcDNA) cells. Columns represent the average of four independent experiments $\square \pm$ SD. $* p<0.05$. c. The amplicon of the GAPDH promoter was used as a negative control. The figure represents four independent experiments. 


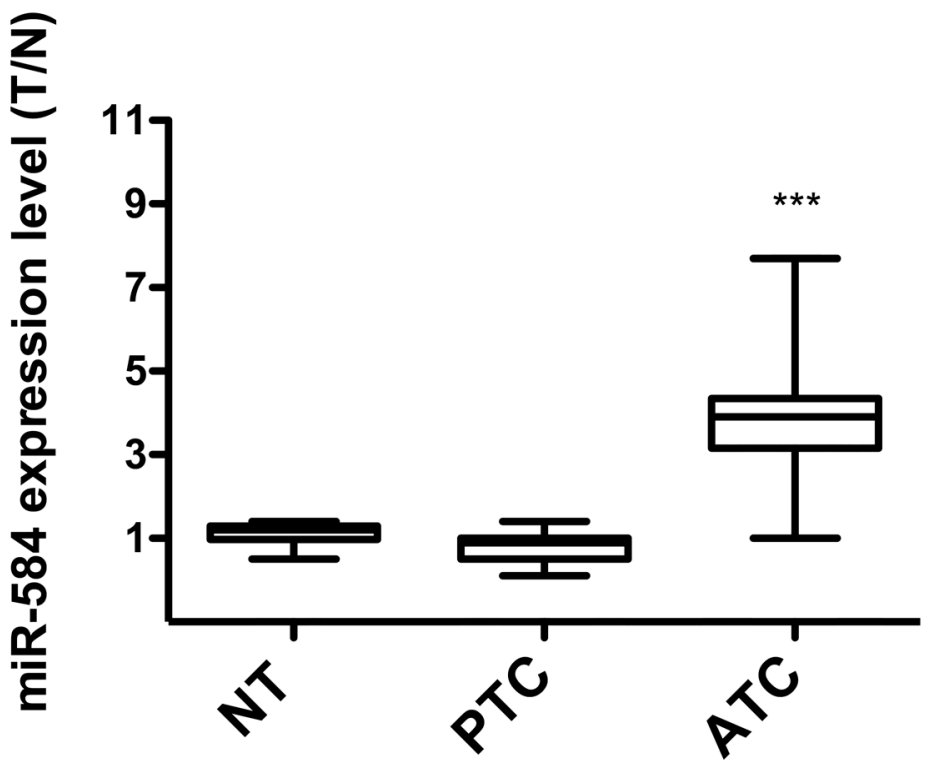

Figure 3: Expression of miR-584 in thyroid tissue samples. qRT-PCR analysis of miR-584 in normal thyroid (NT) (n=10), PTC $(n=11)$, and ATC $(n=6)$ snap-frozen tissue samples. The expression level of miR-584 was normalized to the U6 snRNA levels. The level of miR-584 in each sample was measured by comparing its fluorescence threshold with the average fluorescence threshold of the NT samples. The average results of quadruplicate experiments are plotted. $* * *, p<0.001$.

a

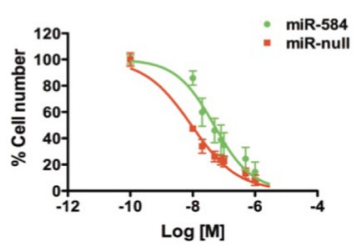

b

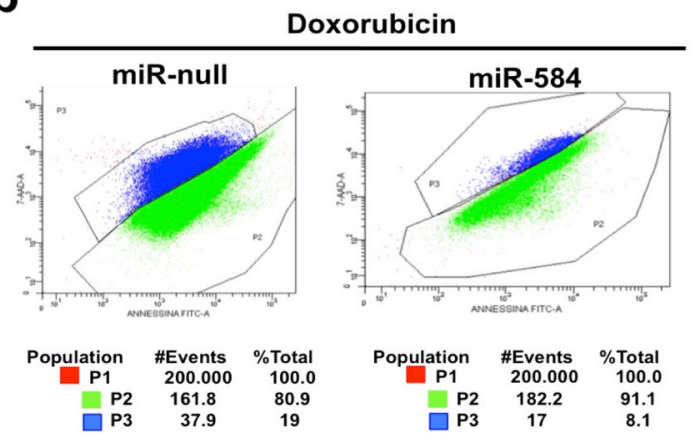

C

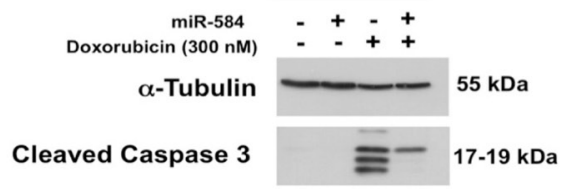

Staurosporine

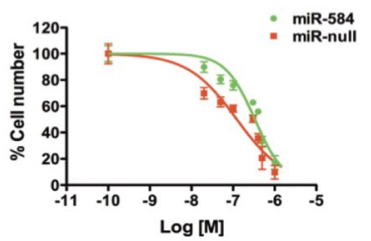

Staurosporine
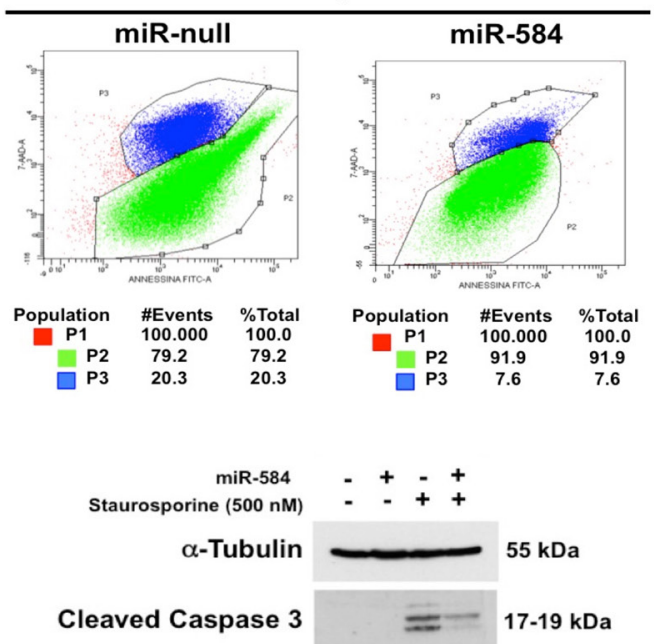

Figure 4: Effects of ectopic expression of miR-584 in TPC cells. a. TPC miR-584 and TPC miR-null cell lines weretreated with

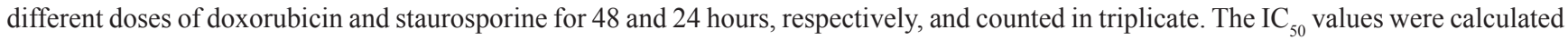
with GraphPad software. The average of three independent experiments \pm SD is shown. b. Dual staining of apoptotic cells with 7-AAD and Annexin V after treatment with doxorubicin and staurosporine. The percentages of apoptotic cells (P3) or of viable cells (P2) are indicated. c. TPC miR-584 and TPC miR-null cells were treated with $300 \mathrm{nM}$ of doxorubicin or with $500 \mathrm{nM}$ of staurosporine, lysed after 48 and 24 hours, respectively, and blotted with the antibodies for Cleaved CASPASE 3 or $\alpha$-TUBULIN. 


\section{miR-584 silencing in TPC-TWIST1 and 8505C cells increases sensitivity to apoptosis induced by doxorubicin and staurosporine}

To confirm these data, we performed the mirror experiment; thus, we stably silenced TPC-TWIST1 cells with anti-miR-584 or a control (anti-null). The silencing efficiency is shown in Figure S3a. miR-584 silencing in TPC-TWIST1 cells did not impair cell proliferation (Figure S3b), migration (Figure S3c) and invasion (Figures S3d and S3e). Then, we treated TPC-TWIST1 anti-miR-584 and anti-null cells with different doses of doxorubicin and staurosporine. As shown in Figure 5a and $5 \mathrm{~b}$, silencing of miR-584 in TPC-TWIST1 cells increased sensitivity to apoptosis. Then, we assessed whether silencing of miR-584 increases sensitivity to apoptosis in an ATC cell line characterized by a more aggressive phenotype. For this aim, we stably transfected $8505 \mathrm{C}$ cells harboring a BRAF V600E mutation, which expressed high endogenous levels of miR-584 (Figure S4a), with antimiR-584 plasmid or control (anti- null). The silencing efficiency is shown in Figure S4b. Silencing of miR-584 in $8505 \mathrm{C}$ cells did not significantly affect cell migration, invasion and proliferation (Figure S5) but increased sensitivity to apoptosis following the treatments (Figure $5 \mathrm{c}$ and 5d), confirming the results obtained in TPC-TWIST1 cells.

\section{TUSC2 is a direct target of miR-584}

miRNAs are negative regulators of gene expression. Using the bioinformatics algorithm TargetScan, we selected TUSC2 (tumor suppressor candidate 2) among the top predicted targets of miR-584 for further analysis. This prediction was also confirmed using other bioinformatics programs (PicTar, DIANA Lab, PITA, RNA22, RNAhybrid, miRDB and miRanda). TUSC2 is down-regulated in several human cancers and induces apoptosis [31]. Initially, we evaluated TUSC2 expression in TPC miR-584 and in TPC-TWIST1 anti-miR-584 cells and controls. As shown in Figure 6a, TUSC2 protein levels were decreased in TPC miR-584 cells compared to TPC miR-null cells. TUSC2 was also down-regulated at the mRNA level (Figure 6b), suggesting that miR-584 both reduced translation and accelerated mRNA degradation of TUSC2. Similarly, TUSC2 protein and mRNA levels were increased compared to the controls in TPC-TWIST1 antimiR-584 cells (Figure 6c and 6d).

To confirm that miR-584 directly regulates TUSC2, we performed a luciferase reporter assay in which we transfected TPC miR-584 and TPC miR-null cells with a vector containing a luciferase reporter gene fused with the $3^{\prime}$ untranslated (UTR) region of TUSC2, which was driven by a constitutive promoter. We also transfected these cells with a mutant version of this plasmid, where the predicted miR-584 binding site of TUSC2 was deleted (Figure 6e). As shown in Figure 6f, the luciferase activity was decreased after transfection of a 3'UTR vector containing TUSC 2 wild type (3'UTR WT). This was reversed when the putative miR-584 binding site in the TUSC2 3 '-UTR (3'UTR del) was deleted (Figure 6f).

\section{TUSC2 rescues the resistance to apoptosis induced by miR-584}

To verify if TUSC2 is involved in resistance to apoptosis induced by miR-584, we transiently transfected TPC miR-584 cells with a construct that expresses TUSC2 but lacks the 3'UTR of the TUSC2-encoding mRNA (thus, the TUSC2 mRNA is refractory to inhibition of translation induced by miR-584) or with a control vector (CMV). Efficiency of transient transfection is shown in Figure 7a. As shown in Figure 7b, TPC miR-584 cells transfected with TUSC2 and treated with doxorubicin $(50 \mathrm{nM})$ or staurosporine $(300 \mathrm{nM})$ showed decreased cell viability compared with control cells (CMV). Thus, TUSC2 partially rescued the phenotype induced by miR-584 in TPC cells. Overall, these results demonstrate that TUSC2 is a functional target of miR-584.

\section{TUSC2 is down-regulated in thyroid carcinoma samples}

Finally, we evaluated the expression level of TUSC2 in 37 normal thyroid (NT), 20 PTC and 40 ATC samples using immunohistochemistry. As shown in Table 1 , all normal thyroids were positive for TUSC2, with $\sim 60 \%$ of the samples showing strong TUSC 2 staining. In contrast, TUSC 2 was undetectable in almost all ATC samples. The majority of PTC samples were negative for TUSC2, suggesting a correlation between TUSC2 downregulation and tumor progression. Representative images of TUSC2 staining in PTC, ATC and normal contralateral thyroid lobes are shown in Figure 8a. We also evaluated TUSC 2 mRNA levels by qRT-PCR in an independent set of samples including 10 NT, 11 PTC and 6 ATC. TUSC2 mRNA was down-regulated in the majority of ATC and in a fraction of PTC samples (Figure 8b).

\section{DISCUSSION}

miRNA targets of Twist1 have not been fully elucidated. Here, we identified a set of potential miRNAs targets of Twist 1 in thyroid cancer cells.

Since deregulation of miRNAs has been described in thyroid carcinogenesis [19, 32, 33], we searched in our array of TPC-TWIST1 cells miRNAs previously reported to be deregulated in thyroid carcinoma samples. We found a decrease of miR-301a and miR-200c in TPC-TWIST1 cells (Table S2); these miRNAs were reported to be downregulated in ATC [34]. 
Additionally, we found that miR-145 and miR-101 were up-regulated in TPC-TWIST1 cells; these miRNAs, in contrast, were down-regulated in ATC samples [3438]. This difference could be explained by the fact that TPC-TWIST1 is a cell line and thus not completely representative of cancer complexity.

Consistent with our findings, miR-584 was also shown to be up-regulated in a microarray screening of different malignant thyroid tissues compared to benign thyroid lesions [39]. Furthermore, Xiang recently reported that transient forced expression of miR-584 in K1 cells had no effect on cell proliferation but decreased cell migration [40]. It is possible that the different results obtained for cell migration compared to our study is due to the different cell lines used and to different transfection methods.

Here, we found that miR-584 was up-regulated in ATC, while it is down-regulated in glioma, where it suppress cell growth [41]; in renal cell carcinoma, where it targets $R O C K 1$ to decrease invasion [42]; and in breast carcinoma [43]. The differential expression of miR-584 in several human cancers may be because the functions of miRNAs are dependent on cellular context, which is due to the different expression of their targets mRNAs.
Accordingly, some miRNAs are oncogenic and upregulated in one cancer type but are tumor suppressors and down-regulated in another cancer type [30].

Using different computational programs, we identified TUSC2 as a novel target of miR-584. TUSC2 is located on chromosome $3 \mathrm{p} 21.3$, a region in which deficient gene expression is observed in lung cancers $[44,45]$. In human lung cancer cell lines, TUSC2 induced apoptosis [46]. Overexpression of TUSC2 inhibited tumor growth and progression in mouse models [47], while TUSC2 knockout mice showed an increased frequency of spontaneous cancers [48, 49].

Here, we showed that TUSC2 partially reverts the phenotype induced by miR-584; thus, it is likely that other targets mediate the effects of miR-584 in TPC cells. Indeed, it is well known that miRNAs act by inhibiting multiple targets.

Moreover, we demonstrated by immunohistochemistry analysis that TUSC2 was downregulated not only in ATC but also in a percentage of PTC samples, which did not express miR-584. This suggests that other mechanisms could control the expression of TUSC2 in PTC samples where TUSC2 is down-

\section{TPC-TWIST1}

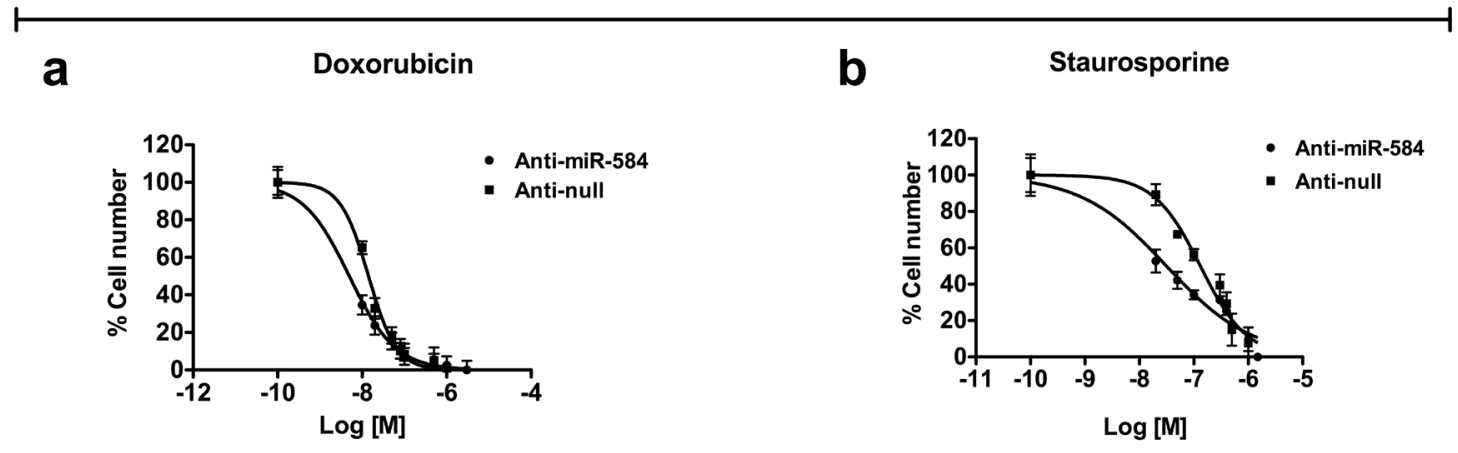

$8505 \mathrm{C}$

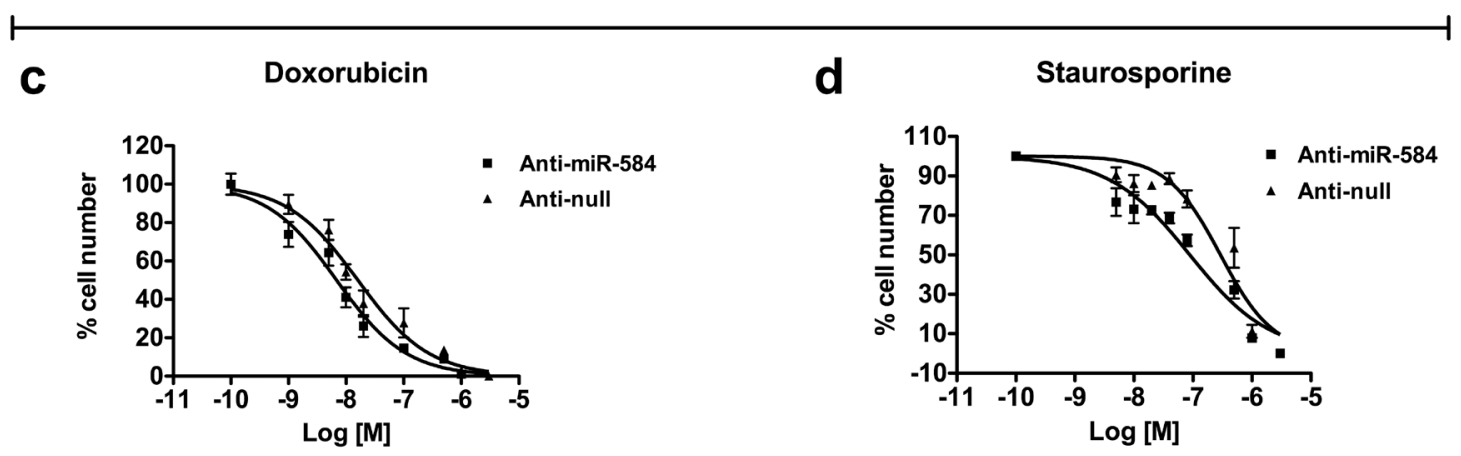

Figure 5: Effects of silencing of miR-584 in TPC-TWIST1 and 8505C cells. The indicatedcells were treated with increasing doses of doxorubicin or staurosporine and counted after 48 and 24 hours, respectively. Values represent the average of four independent experiments $\pm \mathrm{SD}$. 
a

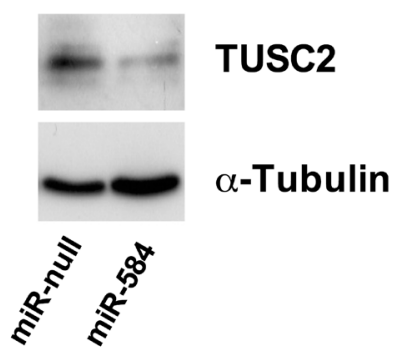

C

TPC-Twist1

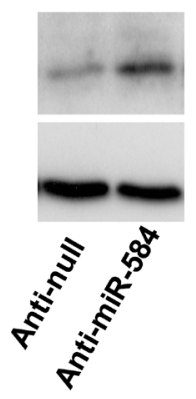

TUSC2

$\alpha-$ Tubulin

e

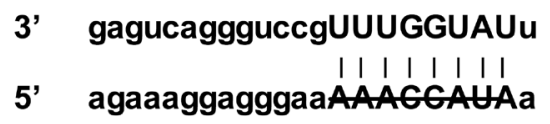

f

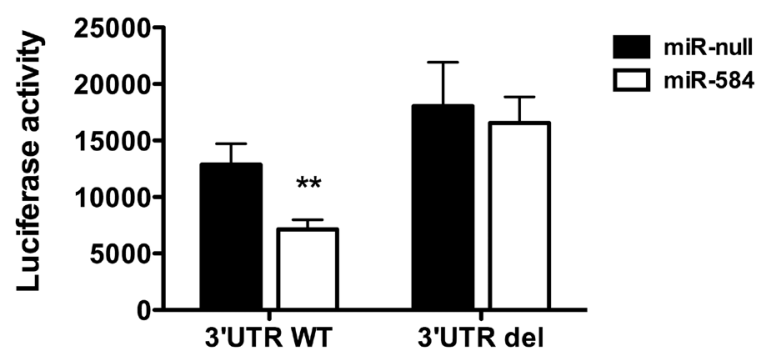

b

TPC

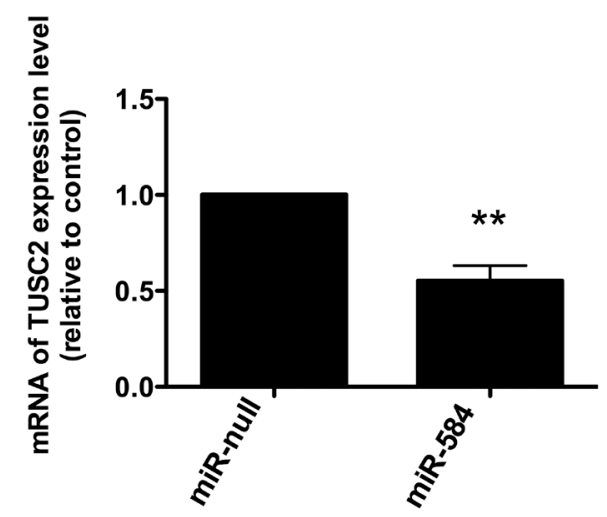

d

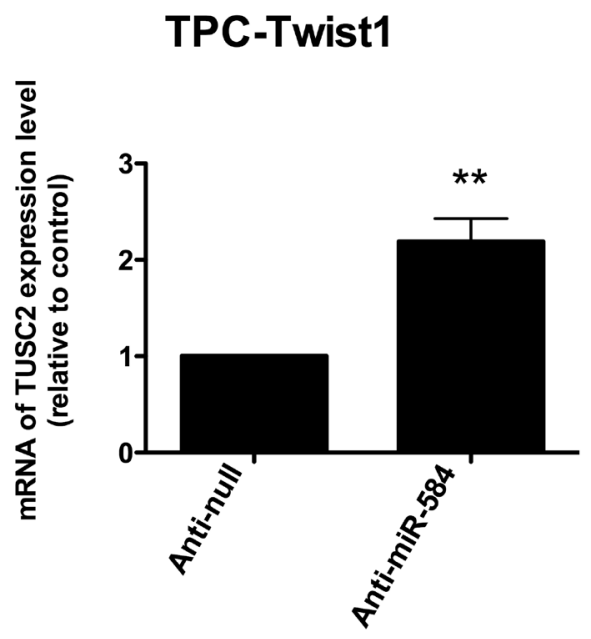

5' hsa-miR-584

3' 3'UTR - TUSC2

Figure 6: TUSC2 is a direct target of miR-584. a. Immunoblotting of TUSC 2 and $\alpha$-TUBULIN in TPC miR-584 or control cells. b. qRT-PCR of TUSC2 in TPC miR-584 or control cells; data were normalized to the level of POLR2A assuming that the value of TPC miRnull is equal to 1 . c. Immunoblotting of TUSC2 and $\alpha$-TUBULIN in TPC-TWIST1 anti-miR-584 or control cells. d. qRT-PCR of TUSC2 in TPC-TWIST1 anti-miR-584 or control cells. e. Predicted duplex formation between human 3'UTR-TUSC2 and miR-584;the strikethrough sequence of 3'UTR of TUSC2 corresponds to the predicted binding site of miR-584 deleted in the mutant 3'UTR-TUSC2 plasmid (3'UTR del). f. Luciferase activity of TUSC2 wild-type (3'UTR WT) or deleted (3'UTR del) reporter genes in TPC cells stably transfected with miR-584 or control. Luciferase activity was normalized with a co-transfected $\beta$-actin plasmid. ${ }^{* *}, p<0.01$. 
regulated. Consistent with this hypothesis, recent data revealed several mechanisms of regulation of TUSC2: different miRNAs (named miR-98, -93 -197 and -378) have been identified as negative regulators of TUSC2 [50,
51]; methylation of TUSC2 was identified in head and neck tumors [52]; myristoylation of the TUSC2 protein has been reported in lung cancers [53]; and two TUSC2

a

\section{$\alpha-$ Tubulin}

\section{TUSC2}

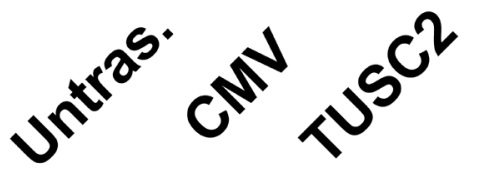

b

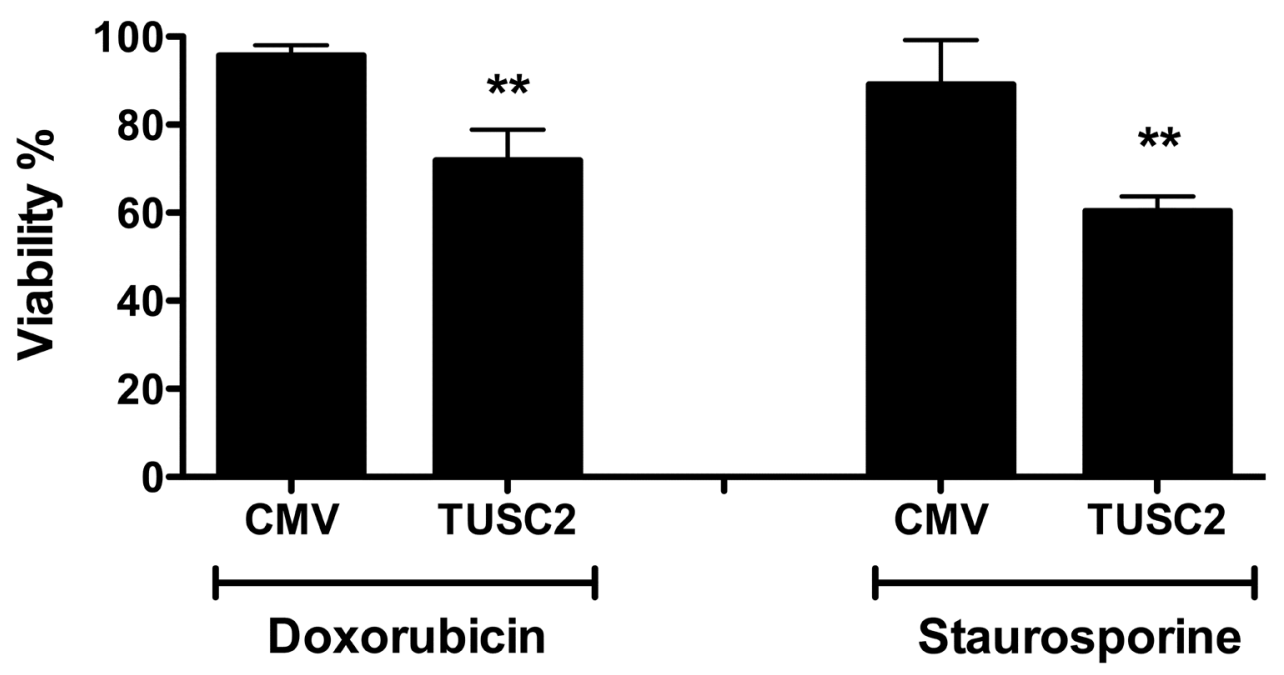

Figure 7: TUSC2 partially rescues the phenotype induced by miR-584. a. TPC miR-584 cells were transiently transfected with TUSC2 plasmid or with the vector control (CMV) and analyzed for TUSC2 protein expression by Western blotting. An antia-TUBULIN antibody was used for normalization. b. TPC miR-584 cells were transfected with TUSC2 or vector controlandtreated with $50 \mathrm{nM}$ of doxorubicin and $300 \mathrm{nM}$ of staurosporine; after 48 and 24 hours, respectively, cells were stained with trypan blue and counted in triplicate. The percentage viability $\pm \mathrm{SD}$ is shown, ${ }^{* *}, p<0.01$. 
Table 1: TUSC2 protein expression in thyroid samples $(n=97)$

\begin{tabular}{|c|c|c|c|}
\hline TUSC2 & $\begin{array}{c}\text { NT } \\
(\mathbf{N}=\mathbf{3 7})\end{array}$ & $\begin{array}{c}\text { PTC } \\
(\mathrm{N}=\mathbf{2 0})\end{array}$ & $\begin{array}{c}\text { ATC } \\
(N=40)\end{array}$ \\
\hline+++ & $22(59.5 \%)$ & - & - \\
\hline++ & $11(29.8 \%)$ & $1(5 \%)$ & - \\
\hline+ & $4(10.8 \%)$ & $4(20 \%)$ & $1(2.5 \%)$ \\
\hline Negative & - & $15(75 \%)$ & $39(97.5 \%)$ \\
\hline
\end{tabular}

,$+ \geq 5$ to $\leq 25 \%$ of positive cells;,$++>25$ to $60 \%$ of positive cells; $+++\geq 60 \%$ of positive cells.

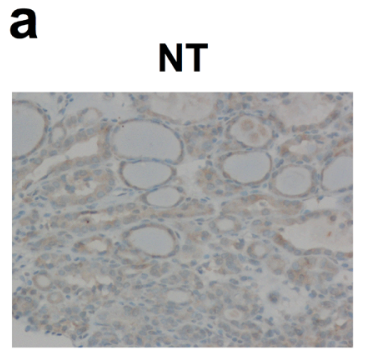

NT

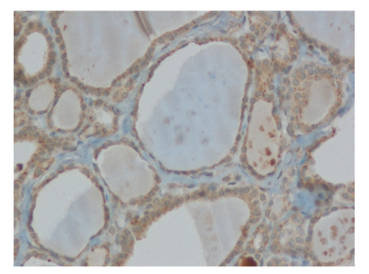

\section{PTC}

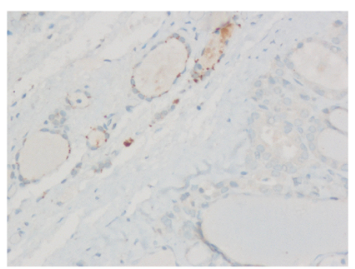

ATC

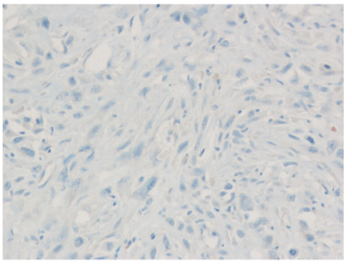

b

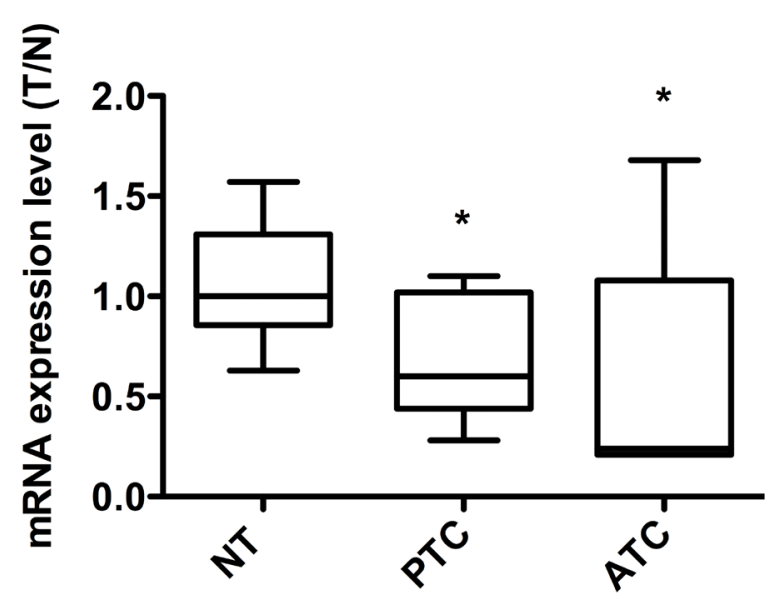

Figure 8: Expression of TUSC2 in thyroid tissue samples. a. Immunohistochemical analysis of TUSC2 protein expression in normal and malignant thyroid tissues. Representative sections (20X magnification) of PTC, ATC and contralateral NT stained with an antiTUSC 2 antibody are shown. b. qRT-PCR of TUSC 2 in NT $(n=10)$, PTC $(n=11)$ and ATC $(n=6)$ snap-frozen tissue samples. The expression level of TUSC2 in each sample was measured by comparing its fluorescence threshold with the average fluorescence threshold of the NT samples. The average results of quadruplicate samples are plotted. The expression level in each sample was normalized with the $\beta$-Actin measurement. $* p<0.05$. 
pseudogenes were identified as regulators of TUSC2 activities [54].

Importantly, TUSC2-expressing nanoparticles have been tested in clinical trials in patients with lung cancer and showed anti-tumor activity and no significant side effects [55]. New therapeutic approaches for treating ATC are needed. Our data suggest that miR-584 and TUSC2 could be used as therapeutic targets for ATC and as novel biomarkers of therapeutic response.

\section{MATERIALS AND METHODS}

\section{Cell culture}

The TPC-1 (named TPC) cell line was obtained from M. Nagao (Carcinogenesis Division, National Cancer Center Research Institute, Tokyo, Japan), and the $8505 \mathrm{C}$ cell line was purchased from DSMZ (Deutsche Sammlung von Mikroorganismen und Zellkulturen $\mathrm{GmbH}$, Braunschweig, Germany). TPC and $8505 \mathrm{C}$ cells were grown in Dulbecco's modified Eagle's medium (DMEM) (Thermo Scientific, Waltham, MA, USA) containing 10\% fetal bovine serum (FBS), L-glutamine and penicillin/ streptomycin (Thermo Scientific). The non-transformed human thyroid follicular epithelial cell line Nthy-ori 3-1 was purchased from Sigma-Aldrich (St. Louis, MO, USA) and grown in RPMI-1640 medium supplemented with 10\% FBS (Thermo Scientific). The cell lines were authenticated by short-tandem repeat profiling performing by BMR Genomics (http://www.bmr-genomics.it) in 2015. TPC-TWIST1 cells (mp1, mp2, and Cl2) were generated and characterized previously [23]. TPC-TWIST1 mp1 was used in all the experiments.

\section{Tissue samples}

Tumors and normal thyroid tissue samples used for immunohistochemical analysis and quantitative RT-PCR were obtained from the Department of molecular, medical, surgical pathology and of critical areas, University of Pisa (Italy). Informed consent was obtained from each subject. The clinicopathological information for the PTC samples is listed in Table S3.

\section{Immunohistochemistry analysis}

Immunohistochemical analysis of TUSC2 expression was performed on formalin-fixed, paraffinembedded (FFPE) tumor sections of 60 tumor cases, including 40 ATC and 20 PTC samples. As a control, thyroid tissues from 8 normal glands of patients who underwent surgery for head and neck tumors and 29 normal contralateral tissues were stained. Rabbit polyclonal anti-human TUSC2 antibody (Proteintech Group, Inc. Chicago, USA) was used (dilution 1:150). Sections were stained using a Ventana automated slide stainer (Ventana Medical Systems, Tucson, Az). TUSC2 expression in epithelial tumor and normal tissue cells was evaluated independently by two investigators (C.U. and F.B.) who were blinded to the clinicopathological data. Discrepancies between the two observers were discussed with a third pathologist (A.P.). For all cases, the percentage of positive cells per 5 high power fields (40X) was determined.

\section{Microarray analysis}

Total RNA was extracted from TPC-TWIST1 (TWIST1 mp1, TWIST1 mp2, TWIST1 Cl2) and pcDNA control cells. miRNA analysis was performed using TaqMan ${ }^{\circledR}$ LDA Cards (Thermo Scientific, Waltham, MA, USA) according to standard procedures and conducted at Aarhus Biotechnology (Aarhus, Denmark).

\section{RNA extraction and qRT-PCR}

Total RNA was isolated using the mirVana ${ }^{\mathrm{TM}}$ miRNA Isolation Kit (Thermo Scientific, Waltham, MA, USA) according to the manufacturer's instructions. RNA samples were quantified using a NanoDrop spectrophotometer (Thermo Scientific) and verified by a 2100 Bioanalyzer (Agilent Technologies, Santa Clara, CA, USA); only samples with RNA integrity number (RIN) values $>7$ were used for further analysis.

For miRNA detection, specific primers and probes (Thermo Scientific) were used. Briefly, $10 \mathrm{ng}$ of total RNA was reverse transcribed using a miRNA Reverse Transcription Kit (Thermo Scientific), followed by amplification using a TaqMan Universal Master Mix II (Thermo Scientific). U6 snRNA was used as an endogenous control.

For gene expression analysis, the mRNA level of TUSC2 was measured by quantitative PCR assays using specific primers and probes (Thermo Scientific) and TaqMan Universal PCR Master Mix (Thermo Scientific). $\beta$-ACTIN and POLR $2 A$ were used as endogenous controls for tumor samples and cell lines, respectively. PCR reactions were performed in triplicate, and fold changes

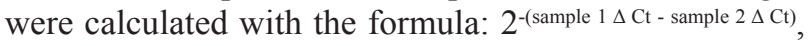
where $\Delta \mathrm{Ct}$ is the difference between the amplification fluorescence thresholds of the gene of interest and the endogenous control used as an internal reference.

\section{Chromatin immunoprecipitation assay}

Chromatin immunoprecipitation (ChiP) assays were performed according to the instructions of the ChiP Assay Kit (Merck, Billerica, MA, USA) and as previously described [25]. Briefly, TPC-pcDNA and TPC-TWIST1 cell lysates were sonicated on ice 30 times for 30 seconds each at the maximum settings. Samples were subjected to immunoprecipitation with anti-TWIST1 antibody 
(Twist2C1a, sc-81417; Santa Cruz Biotechnology, Dallas, USA) or control antibody (mouse IgG1 isotype control, clone G3A1; Cell Signaling Technology, Beverly, USA). For qRT-PCR, 2 of $30 \mu \mathrm{L}$ immunoprecipitated DNA was used. Input DNA values were used to normalize the values from the quantitative ChIP samples. Percent input was calculated as $2^{-\Delta \mathrm{Ct} x}$, where $\mathrm{Ct}$ is cycle threshold and $\Delta \mathrm{Ct}$ is the difference between $\mathrm{Ct}$ of input and $\mathrm{Ct}$ of immunoprecipitated samples. ChIP values represent the average of three independent experiments, and for each experiment qRT-PCR was performed in triplicate. The GAPDH promoter amplicon was used as a negative control. The sequences of the primers covering E-box regions used in ChIP assays were as follows:

miR-584 Forward Primer: 5'-tgcaatgtgtgtgttagcca-3'; miR-584 Reverse Primer: 5'-atcattgctccttggctggt-3'; GAPDH Forward Primer: 5'-cccaaagtcctcctgtttca-3'; GAPDH Reverse Primer: 5'-gtcttgaggcctgagctac-3'.

\section{Cell transfections}

The miR-584 precursor construct expressing premiR-584 (pEP-hsa-miR-584) and the corresponding empty vector ( $\mathrm{pEP}$-miR, named miR-null) were purchased from Cell Biolabs (San Diego, USA). For miRNA silencing, the hsa-miR-584 inhibitor and control vector (named antimiR-584 and anti-miR-null, respectively) were purchased from GeneCopoeia (Labomics, Nivelles, Belgium).

To generate stable transfectants $1 \times 10^{5}$ cells were incubated with $50 \mathrm{nmol} / \mathrm{ml}$ of plasmid (pEP-hsa-miR-584 or hsa-miR-584 inhibitor) or with their respective controls and transfected using Lipofectamine 2000 (Thermo Scientific) according to the manufacturer's instructions. After 48 hours, cell populations and clones were selected in $1 \mathrm{mg} / \mathrm{ml}$ puromycin (Sigma-Aldrich, St. Louis, MO, USA) and analyzed for miR-584 expression by qRT-PCR. One cell mass population was selected and used in all the experiments.

For transient transfection, a TUSC2 plasmid, which expresses TUSC2 but lacks the 3'UTR of TUSC2encoding mRNA, and a scrambled control (named CMV) were purchased from GeneCopoeia (Nivelles, Belgium). TPC miR-584 cells were transiently transfected using Lipofectamine 2000 according to the manufacturer's instructions. TUSC2 expression was evaluated by Western blot analysis.

\section{Cell proliferation, migration and invasion assays}

For cell proliferation rate, $5 \times 10^{4}$ cells were plated in 6-well plates and counted in triplicate every 24 hours for 4 days.

Cell migration and invasion, which were assessed by wound-healing and Matrigel matrix (BD Biosciences, San Jose, CA, USA) assays, respectively, were evaluated as previously described [25].

\section{Apoptosis assays}

Cells were plated in triplicate in 6-well plates at a density of $5 \times 10^{5}$ cells and kept in DMEM supplemented with $10 \%$ FBS. The day after plating, the medium was replaced by fresh medium with or without various concentrations of doxorubicin (10 nM, $20 \mathrm{nM}, 50 \mathrm{nM}, 80$ $\mathrm{nM}, 100 \mathrm{nM}, 0.5 \mu \mathrm{M}, 1 \mu \mathrm{M}, 3 \mu \mathrm{M}$ ) (Sigma-Aldrich) and staurosporine ( $20 \mathrm{nM}, 50 \mathrm{nM}, 100 \mathrm{nM}, 0.3 \mu \mathrm{M}, 0.4 \mu \mathrm{M}$, $0.5 \mu \mathrm{M}, 1 \mu \mathrm{M}, 1.5 \mu \mathrm{M}$ ) (Sigma-Aldrich). To estimate the $\mathrm{IC}_{50}$ value, cells were counted with a $\mathrm{TC} 10^{\mathrm{TM}}$ Automated Cell Counter (Bio-Rad, Richmond, VA, USA) after 24 or 48 hours of treatment with staurosporine and doxorubicin, respectively. $\mathrm{IC}_{50}$ values were calculated using GraphPad Prism software (La Jolla, CA, USA).

For determination of cell viability, cells were collected by trypsinization, stained for $10 \mathrm{~min}$ with $0.4 \%$ trypan-blue (Sigma-Aldrich) according to manufacturer's instructions, and counted in triplicate.

Cell death was determined by fluorescence-activated cell sorting (FACS) analysis using 7-amino-actinomycin (7-AAD) for dead cells and Annexin V (Beckman Coulter, Miami, FL, USA) for apoptotic cells according to the manufacturer's protocol. Viable cells stained negative for 7-AAD and Annexin V.

\section{Western blot}

Immunoblotting was carried out according to standard procedures. Membranes were probed with the indicated antibodies: rabbit polyclonal anti-TUSC2 (Proteintech) diluted 1:200; rabbit polyclonal anti-Cleaved (Asp175) CASPASE 3 (Cell Signaling Technology) diluted 1:500; mouse monoclonal anti- $\alpha$ TUBULIN (Sigma-Aldrich) diluted 1:10000. Secondary antimouse and anti-rabbit antibodies coupled to horseradish peroxidase were purchased from Bio-Rad and diluted 1:3000. Immune complexes were developed by an enhanced chemiluminescence detection kit (Thermo Scientific).

\section{Luciferase reporter assay}

TPC miR-584 cells were seeded into a 96-well plate $\left(1 \times 10^{3}\right.$ cells per well) and kept in DMEM supplemented with $10 \%$ FBS. After 24 hours, the pLightSwitch 3'UTR reporter plasmids (pLightSwitch-TUSC2-3'UTR or pLightSwitch-Empty-3'UTR and pLightSwitch-TUSC23'UTR del) were transfected into the cells using FuGENE Transfection Reagent (Roche, Basel, Switzerland) according to the manufacturer's protocol. Luciferase activity was measured 24 hours after transfection using the LightSwitch Luciferase Assay reagent (SwitchGear Genomics, CA, USA) according to the manufacturer's protocol.

Deletion of the 3'UTR of TUSC2 was introduced in the pLightSwitch-TUSC2-3'UTR WT plasmid using 
the QuikChange site-directed mutagenesis kit (Agilent Technologies). The following oligonucleotides were used: TUSC2-3'UTR del Forward: 5'GGGACTGTTCACCACCTTGT- 3'; TUSC2-3'UTR del Reverse: 5'CCCAAGCCATTTCCCACATT-3'.

\section{Statistical analysis}

Results were analyzed with unpaired t tests using GraphPad Prism 5 software (La Jolla, CA, USA) and are shown as the mean \pm standard deviation (SD). Differences were considered significant when $p<0.05$.

\section{ACKNOWLEDGMENTS}

We thank Massimo Santoro for continuous support, Giulia Scalia and Maddalena Raia for assistance with the flow cytometry analysis and Luigi Auletta for assistance with the statistical analysis. This work was supported by the Italian Association for Cancer Research (IG-11885) and the Italian Ministry of Health (GR-2010 -2314003).

\section{CONFLICTS OF INTEREST}

The authors declare no conflicts of interest.

\section{REFERENCES}

1. Qin Q, Xu Y, He T, Qin C, Xu J. Normal and disease-related biological functions of Twist1 and underlying molecular mechanisms. Cell research. 2012; 22:90-106.

2. Ansieau S, Morel AP, Hinkal G, Bastid J, Puisieux A. TWISTing an embryonic transcription factor into an oncoprotein. Oncogene. 2010; 29:3173-3184.

3. Wushou A, Hou J, Zhao YJ, Shao ZM. Twist-1 up-regulation in carcinoma correlates to poor survival. Int J Mol Sci. 2014; 15:21621-21630.

4. Aparicio LA, Blanco M, Castosa R, Concha A, Valladares M, Calvo L, Figueroa A. Clinical implications of epithelial cell plasticity in cancer progression. Cancer letters. 2015; 366:1-10.

5. Eckert MA, Lwin TM, Chang AT, Kim J, Danis E, OhnoMachado L, Yang J. Twist1-induced invadopodia formation promotes tumor metastasis. Cancer cell. 2011; 19:372-386.

6. Schmidt JM, Panzilius E, Bartsch HS, Irmler M, Beckers J, Kari V, Linnemann JR, Dragoi D, Hirschi B, Kloos UJ, Sass S, Theis F, Kahlert S, Johnsen SA, Sotlar K, Scheel $\mathrm{CH}$. Stem-cell-like properties and epithelial plasticity arise as stable traits after transient Twist1 activation. Cell reports. 2015; 10:131-139.

7. Antoniou A, Hebrant A, Dom G, Dumont JE, Maenhaut C. Cancer stem cells, a fuzzy evolving concept: a cell population or a cell property? Cell cycle (Georgetown, Tex). 2013; 12:3743-3748.
8. Maestro R, Dei Tos AP, Hamamori Y, Krasnokutsky S, Sartorelli V, Kedes L, Doglioni C, Beach DH, Hannon GJ. Twist is a potential oncogene that inhibits apoptosis. Genes \& development. 1999; 13:2207-2217.

9. Nikiforov YE, Nikiforova MN. Molecular genetics and diagnosis of thyroid cancer. Nature reviews Endocrinology. 2011; 7:569-580.

10. Xing M. Molecular pathogenesis and mechanisms of thyroid cancer. Nature reviews Cancer. 2013; 13:184-199.

11. Integrated genomic characterization of papillary thyroid carcinoma. Cell. 2014; 159:676-690.

12. Smallridge RC, Ain KB, Asa SL, Bible KC, Brierley JD, Burman KD, Kebebew E, Lee NY, Nikiforov YE, Rosenthal MS, Shah MH, Shaha AR, Tuttle RM. American Thyroid Association guidelines for management of patients with anaplastic thyroid cancer. Thyroid. 2012; 22:1104-1139.

13. Haddad RI, Lydiatt WM, Ball DW, Busaidy NL, Byrd D, Callender G, Dickson P, Duh QY, Ehya H, Haymart M, Hoh C, Hunt JP, Iagaru A, et al. Anaplastic Thyroid Carcinoma, Version 2.2015. Journal of the National Comprehensive Cancer Network: JNCCN. 2015; 13:1140-1150.

14. Ragazzi M, Ciarrocchi A, Sancisi V, Gandolfi G, Bisagni A, Piana S. Update on anaplastic thyroid carcinoma: morphological, molecular, and genetic features of the most aggressive thyroid cancer. International journal of endocrinology. 2014; 2014:790834.

15. Hsu KT, Yu XM, Audhya AW, Jaume JC, Lloyd RV, Miyamoto S, Prolla TA, Chen H. Novel approaches in anaplastic thyroid cancer therapy. The oncologist. 2014; 19:1148-1155.

16. Lee DY, Won JK, Lee SH, Park DJ, Jung KC, Sung MW, Wu HG, Kim KH, Park YJ, Hah JH. Changes of Clinicopathologic Characteristics and Survival Outcomes of Anaplastic and Poorly Differentiated Thyroid Carcinoma. Thyroid. 2015.

17. Smith N, Nucera C. Personalized therapy in patients with anaplastic thyroid cancer: targeting genetic and epigenetic alterations. The Journal of clinical endocrinology and metabolism. 2015; 100:35-42.

18. Caillou B, Talbot M, Weyemi U, Pioche-Durieu C, Al Ghuzlan A, Bidart JM, Chouaib S, Schlumberger M, Dupuy C. Tumor-associated macrophages (TAMs) form an interconnected cellular supportive network in anaplastic thyroid carcinoma. PloS one. 2011; 6:e22567.

19. Hebrant A, Floor S, Saiselet M, Antoniou A, Desbuleux A, Snyers B, La C, de Saint Aubain N, Leteurtre E, Andry G, Maenhaut C. miRNA expression in anaplastic thyroid carcinomas. PloS one. 2014; 9: e103871.

20. Jung CW, Han KH, Seol H, Park S, Koh JS, Lee SS, Kim MJ, Choi IJ, Myung JK. Expression of cancer stem cell markers and epithelial-mesenchymal transition-related factors in anaplastic thyroid carcinoma. International journal of clinical and experimental pathology. 2015; 8:560-568. 
21. Buehler D, Hardin H, Shan W, Montemayor-Garcia C, Rush PS, Asioli S, Chen H, Lloyd RV. Expression of epithelialmesenchymal transition regulators SNAI2 and TWIST1 in thyroid carcinomas. Mod Pathol. 2013; 26:54-61.

22. Montemayor-Garcia C, Hardin H, Guo Z, Larrain C, Buehler D, Asioli S, Chen H, Lloyd RV. The role of epithelial mesenchymal transition markers in thyroid carcinoma progression. Endocrine pathology. 2013; 24:206-212.

23. Salerno P, Garcia-Rostan G, Piccinin S, Bencivenga TC, Di Maro G, Doglioni C, Basolo F, Maestro R, Fusco A, Santoro M, Salvatore G. TWIST1 plays a pleiotropic role in determining the anaplastic thyroid cancer phenotype. The Journal of clinical endocrinology and metabolism. 2011; 96:E772-781.

24. Hardy RG, Vicente-Duenas C, Gonzalez-Herrero I, Anderson C, Flores T, Hughes S, Tselepis C, Ross JA, Sanchez-Garcia I. Snail family transcription factors are implicated in thyroid carcinogenesis. The American journal of pathology. 2007; 171:1037-1046.

25. Di Maro G, Orlandella FM, Bencivenga TC, Salerno P, Ugolini C, Basolo F, Maestro R, Salvatore G. Identification of targets of Twist 1 transcription factor in thyroid cancer cells. The Journal of clinical endocrinology and metabolism. 2014; 99:E1617-1626.

26. Yin G, Chen R, Alvero AB, Fu HH, Holmberg J, Glackin C, Rutherford T, Mor G. TWISTing stemness, inflammation and proliferation of epithelial ovarian cancer cells through MIR199A2/214. Oncogene. 2010; 29:3545-3553.

27. Drasin DJ, Guarnieri AL, Neelakantan D, Kim J, Cabrera JH, Wang CA, Zaberezhnyy V, Gasparini P, Cascione L, Huebner K, Tan AC, Ford HL. TWIST1-Induced miR424 Reversibly Drives Mesenchymal Programming while Inhibiting Tumor Initiation. Cancer research. 2015; 75:1908-1921.

28. Lee YB, Bantounas I, Lee DY, Phylactou L, Caldwell MA, Uney JB. Twist-1 regulates the miR-199a/214 cluster during development. Nucleic acids research. 2009; 37:123-128.

29. Ma L, Teruya-Feldstein J, Weinberg RA. Tumour invasion and metastasis initiated by microRNA-10b in breast cancer. Nature. 2007; 449:682-688.

30. Di Leva G, Garofalo M, Croce CM. MicroRNAs in cancer. Annual review of pathology. 2014; 9:287-314.

31. Uzhachenko R, Shanker A, Yarbrough WG, Ivanova AV. Mitochondria, calcium, and tumor suppressor Fus1: At the crossroad of cancer, inflammation, and autoimmunity. Oncotarget. 2015; 6:20754-20772. doi: 10.18632/ oncotarget. 4537

32. Fuziwara CS, Kimura ET. MicroRNA Deregulation in Anaplastic Thyroid Cancer Biology. International journal of endocrinology. 2014; 2014:743450.

33. Pallante P, Battista S, Pierantoni GM, Fusco A. Deregulation of microRNA expression in thyroid neoplasias. Nature reviews Endocrinology. 2014; 10:88-101.
34. Braun J, Hoang-Vu C, Dralle H, Huttelmaier S. Downregulation of microRNAs directs the EMT and invasive potential of anaplastic thyroid carcinomas. Oncogene. 2010; 29:4237-4244.

35. Wang $\mathrm{C}, \mathrm{Lu} \mathrm{S}$, Jiang J, Jia X, Dong X, Bu P. HsamicroRNA-101 suppresses migration and invasion by targeting Rac1 in thyroid cancer cells. Oncology letters. 2014; 8:1815-1821.

36. Lin X, Guan H, Li H, Liu L, Liu J, Wei G, Huang Z, Liao Z, Li Y. miR-101 inhibits cell proliferation by targeting Rac1 in papillary thyroid carcinoma. Biomedical reports. 2014; 2:122-126.

37. Boufraqech M, Zhang L, Jain M, Patel D, Ellis R, Xiong Y, He M, Nilubol N, Merino MJ, Kebebew E. miR-145 suppresses thyroid cancer growth and metastasis and targets AKT3. Endocrine-related cancer. 2014; 21:517-531.

38. Gu Y, Li D, Luo Q, Wei C, Song H, Hua K, Song J, Luo Y, Li X, Fang L. MicroRNA-145 inhibits human papillary cancer TPC1 cell proliferation by targeting DUSP6. International journal of clinical and experimental medicine. 2015; 8:8590-8598.

39. Vriens MR, Weng J, Suh I, Huynh N, Guerrero MA, Shen WT, Duh QY, Clark OH, Kebebew E. MicroRNA expression profiling is a potential diagnostic tool for thyroid cancer. Cancer. 2012; 118:3426-3432.

40. Xiang J, Wu Y, Li DS, Wang ZY, Shen Q, Sun TQ, Guan Q, Wang YJ. miR-584 Suppresses Invasion and Cell Migration of Thyroid Carcinoma by Regulating the Target Oncogene ROCK1. Oncology research and treatment. 2015; 38:436-440.

41. Wang XP, Deng XL, Li LY. MicroRNA-584 functions as a tumor suppressor and targets PTTG1IP in glioma. International journal of clinical and experimental pathology. 2014; 7:8573-8582.

42. Ueno K, Hirata H, Shahryari V, Chen Y, Zaman MS, Singh K, Tabatabai ZL, Hinoda Y, Dahiya R. Tumour suppressor microRNA-584 directly targets oncogene Rock-1 and decreases invasion ability in human clear cell renal cell carcinoma. British journal of cancer. 2011; 104:308-315.

43. Fils-Aime N, Dai M, Guo J, El-Mousawi M, Kahramangil B, Neel JC, Lebrun JJ. MicroRNA-584 and the protein phosphatase and actin regulator 1 (PHACTR1), a new signaling route through which transforming growth factorbeta Mediates the migration and actin dynamics of breast cancer cells. The Journal of biological chemistry. 2013; 288:11807-11823.

44. Lerman MI, Minna JD. The 630-kb lung cancer homozygous deletion region on human chromosome 3p21.3: identification and evaluation of the resident candidate tumor suppressor genes. The International Lung Cancer Chromosome 3p21.3 Tumor Suppressor Gene Consortium. Cancer research. 2000; 60:6116-6133.

45. Prudkin L, Behrens C, Liu DD, Zhou X, Ozburn NC, Bekele BN, Minna JD, Moran C, Roth JA, Ji L, Wistuba, II. Loss and reduction of FUS1 protein expression is a frequent 
phenomenon in the pathogenesis of lung cancer. Clinical cancer research. 2008; 14:41-47.

46. Ji L, Roth JA. Tumor suppressor FUS1 signaling pathway. Journal of thoracic oncology. 2008; 3:327-330.

47. Ji L, Nishizaki M, Gao B, Burbee D, Kondo M, Kamibayashi C, Xu K, Yen N, Atkinson EN, Fang B, Lerman MI, Roth JA, Minna JD. Expression of several genes in the human chromosome 3p21.3 homozygous deletion region by an adenovirus vector results in tumor suppressor activities in vitro and in vivo. Cancer research. 2002; 62:2715-2720.

48. Li L, Yu C, Ren J, Ye S, Ou W, Wang Y, Yang W, Zhong G, Chen X, Shi H, Su X, Chen L, Zhu W. Synergistic effects of eukaryotic coexpression plasmid carrying LKB1 and FUS1 genes on lung cancer in vitro and in vivo. Journal of cancer research and clinical oncology. 2014; 140:895-907.

49. Ivanova AV, Ivanov SV, Pascal V, Lumsden JM, Ward JM, Morris N, Tessarolo L, Anderson SK, Lerman MI. Autoimmunity, spontaneous tumourigenesis, and IL-15 insufficiency in mice with a targeted disruption of the tumour suppressor gene Fus1. The Journal of pathology. 2007; 211:591-601.

50. Lee DY, Deng Z, Wang CH, Yang BB. MicroRNA-378 promotes cell survival, tumor growth, and angiogenesis by targeting SuFu and Fus-1 expression. Proceedings of the National Academy of Sciences of the United States of America. 2007; 104:20350-20355.
51. Du L, Schageman JJ, Subauste MC, Saber B, Hammond SM, Prudkin L, Wistuba, II, Ji L, Roth JA, Minna JD, Pertsemlidis A. miR-93, miR-98, and miR-197 regulate expression of tumor suppressor gene FUS1. Molecular cancer research: MCR. 2009; 7:1234-1243.

52. Demokan S, Chuang AY, Chang X, Khan T, Smith IM, Pattani KM, Dasgupta S, Begum S, Khan Z, Liegeois NJ, Westra WH, Sidransky D, Koch W, Califano JA. Identification of guanine nucleotide-binding protein gamma-7 as an epigenetically silenced gene in head and neck cancer by gene expression profiling. International journal of oncology. 2013; 42:1427-1436.

53. Uno F, Sasaki J, Nishizaki M, Carboni G, Xu K, Atkinson EN, Kondo M, Minna JD, Roth JA, Ji L. Myristoylation of the fus1 protein is required for tumor suppression in human lung cancer cells. Cancer research. 2004; 64:2969-2976.

54. Rutnam ZJ, Du WW, Yang W, Yang X, Yang BB. The pseudogene TUSC2P promotes TUSC2 function by binding multiple microRNAs. Nature communications. 2014; 5:2914.

55. Lu C, Stewart DJ, Lee JJ, Ji L, Ramesh R, Jayachandran G, Nunez MI, Wistuba, II, Erasmus JJ, Hicks ME, Grimm EA, Reuben JM, Baladandayuthapani V, Templeton NS, McMannis JD, Roth JA. Phase I clinical trial of systemically administered TUSC2(FUS1)-nanoparticles mediating functional gene transfer in humans. PloS one. 2012; 7:e34833. 